\title{
A New Ultra Fast Conduction Mechanism in Insulating Polymer Nanocomposites
}

\author{
M. Xu, ${ }^{1}$ G. C. Montanari, ${ }^{2}$ D. Fabiani, ${ }^{2}$ L. A. Dissado, ${ }^{3}$ and A. Krivda ${ }^{4}$ \\ ${ }^{1}$ State Key Laboratory of Electrical Insulation and Power Equipment, Xi'an Jiaotong University, West Xianning Road 28, \\ Xi'an 710049, China \\ ${ }^{2}$ Department of Electrical Engineering, University of Bologna, Viale Risorgimento, 2-40136, Bologna, Italy \\ ${ }^{3}$ Department of Engineering, University of Leicester, University Road, Leicester LE17RH, UK \\ ${ }^{4}$ ABB Switzerland Ltd, Corporate Research, CH-5405 Baden-Daettwil, Switzerland
}

Correspondence should be addressed to M.Xu, xumman@mail.xjtu.edu.cn

Received 8 February 2011; Accepted 13 March 2011

Academic Editor: Guifu Zou

Copyright (C) 2011 M. Xu et al. This is an open access article distributed under the Creative Commons Attribution License, which permits unrestricted use, distribution, and reproduction in any medium, provided the original work is properly cited.

\begin{abstract}
A brand new phenomenon, namely, electrical conduction via soliton-like ultra fast space charge pulses, recently identified in unfilled cross-linked polyethylene, is shown for the first time to occur in insulating polymer nanocomposites and its characteristics correlated with the electromechanical properties of nanostructured materials. These charge pulses are observed to cross the insulation under low electrical field in epoxy-based nanocomposites containing nanosilica particles with relative weights of $1 \%$, $5 \%, 10 \%$, and $20 \%$ at speeds orders of magnitude higher than those expected for carriers in insulating polymers. The characteristics of mobility, magnitude and repetition rate for both positive and negative charge pulses are studied in relation to nanofiller concentration. The results show that the ultra fast charge pulses (packets) are affected significantly by the concentration of nanoparticles. An explanation is presented in terms of a new conduction mechanism where the mechanical properties of the polymer and movement of polymer chains play an important role in the injection and transport of charge in the form of pulses. Here, the charge transport is not controlled by traps. Instead, it is driven by the contribution of polarization and the resultant electromechanical compression, which is substantially affected by the introduction of nanoparticles into the base polymer.
\end{abstract}

\section{Introduction}

Insulating polymers are a category of organic materials widely used in electrical apparatus, exhibiting low charge carrier concentration and mobility (usually $10^{-16}$ $10^{-14} \mathrm{~m}^{2} \mathrm{~V}^{-1} \mathrm{~s}^{-1}$ ), thus low electrical conductivity, even at high fields [1]. Carriers in insulating polymers are introduced by impurities and contaminants, as well as by charges injected from the electrode-insulation interface. When the electrical field applied to the polymer is higher than the threshold for space charge accumulation, charge would be injected into the bulk material from the interface of polymerelectrode and accumulate [2-4], forming homocharge or heterocharge. Based on this traditional conduction mechanism, the current is a continuous flow of carriers, which gradually approaches a steady state value at a given time after the onset of voltage application.
Recently, a brand new phenomenon of charge transport was discovered in some insulating polymers (e.g., minicable with polyethylene insulation $[5,6]$ and nanostructured epoxy-based material [7]) at relatively low electrical fields, through the use of an ultra fast space charge acquisition system and a conduction current measurement apparatus endowed of low-pass filter [8]. Charge pulses (packets) are observed to travel very rapidly through the insulation bulk with mobility 4 to 5 orders of magnitude higher than that for conventional conduction. These repetitive charge packets have magnitudes that are almost independent of field and temperature and do not show appreciable pulse distortion and attenuation during transit, thus behaving as solitons. Such a mechanism should be, therefore, different from that responsible for traditional charge transportation by hopping through localized states (traps). Previous research work has indicated that this new conduction mechanism could 
Table 1: Specimen description.

\begin{tabular}{lc}
\hline Specimen & Weight percentage of $\mathrm{Nano}_{-} \mathrm{SiO}_{2}$ in epoxy resin \\
\hline No. 0 & $0 \%$ \\
No. 1 & $1 \%$ \\
No. 5 & $5 \%$ \\
No. 10 & $10 \%$ \\
No. 20 & $20 \%$ \\
\hline
\end{tabular}

be associated with the electromechanical compression of the polymer and polarization mode. It is known that the morphology of a polymer can be modified by nanofillers (e.g., nanoparticles, nanowires, and nanolayers), due to the large interface interaction (surface to volume ratio) [9], so that the mechanical, thermal, and electrical properties of the polymer could be affected significantly by the introduction of nanofillers $[10,11]$.

Here, we report evidence for the presence of such solitonlike ultra fast space charge packets in epoxy-based nanocomposites. The relationship between polymer structure and mechanical property is discussed, and the influence of nanoparticles on the characteristics of the ultra fast charge pulses is investigated in order to determine whether the relationship expected of a mechanism based on electromechanical compression is viable. The experimental results show that the mechanical properties of the nanocomposite material do indeed affect the ultra fast charge packet conduction to a great extent.

\section{Experiments}

2.1. Materials. Flat specimens of pure epoxy resin and nanofilled composites were prepared. The base epoxy resin (bisphenol A) and the nanocomposites were cured by anhydride hardener with flexibilizer $(20 \mathrm{wt} \%)$. The specimens were $1 \mathrm{~mm}$ in thickness. The diameter of the filling particles, nanosilica $\left(\mathrm{SiO}_{2}\right)$, was about $25 \mathrm{~nm}$ with a density of $2.1 \times$ $10^{3} \mathrm{~kg} \mathrm{~m}^{-3}$. The description of the specimens is reported in Table 1.

Epoxy resin and nano- $\mathrm{SiO}_{2}$ (Nanopox masterbatch) were mixed for 5 to 10 minutes at $65^{\circ} \mathrm{C}$ with four blades stainless steel propeller at the speed of $240 \mathrm{r} / \mathrm{min}$. The hardener was added and mixed under the same conditions for another 15 minutes. In the end, the cure accelerator was added into the mixture. The mixture was degassed at about $200 \mathrm{~Pa}$ pressure at $65^{\circ} \mathrm{C}$. Casting was performed into preheated stainless steel moulds. After casting, degassing was performed once again to remove any air bubbles created during the casting process. Curing was carried out at $80^{\circ} \mathrm{C}$ for 4 hours and postcuring was performed at $120^{\circ} \mathrm{C}$ for 10 . The casting parameters used in sample preparation follow procedures recommended by epoxy manufacturers within the permitted range.

2.2. Test Device. Structure of the specimen (nanoparticle dispersion) was observed by Scanning Electron Microscope (SEM) EL20 produced by FEI corporation. The specimen was broken in liquid nitrogen and metallized on the fracture section for observation. Space charge accumulation was investigated through the Pulsed Electro Acoustic (PEA) technique. The measurements were performed under the electrical field of $10 \mathrm{kV} \mathrm{mm}^{-1}$ and a temperature of $70^{\circ} \mathrm{C}$. The ultra fast charge packets were recorded by means of Digital Signal Averager, which can acquire, average, and store PEA signals at a very high rate (up to several $\mathrm{MHz}$ ). Therefore, the space charge profiles were recorded every $0.02 \mathrm{~s}$, each profile being an average of 100 acquired PEA signals.

\section{Results and Discussion}

The dimension and dispersion of nanoparticles in epoxy resin are shown in Figure 1. The observed specimen is no. 10 ( $10 \%$ of nanoparticles). It could be seen that nanoparticles are dispersed evenly in the base polymer with the average diameter less than $80 \mathrm{~nm}$. The interface of nanoparticles and base polymer is not very clear which indicates the particles are coated well by the polymer chains. The abundant microtopography in the fracture section presents the ductile rupture of the specimen. This kind of structure is beneficial to the improvement of mechanical property.

The space charge patterns of pure and nanofilled composites during the first 30 seconds of polarization are shown in Figure 2. The polarity of charge is indicated by different colors, that is, cold colors for negative charge (e.g., black, blue, purple) and warm colors for positive charge (e.g., red, yellow, green). Hence, the distribution, density, and dynamic of the charge can be found in the figure reporting the charge pattern. Heterocharge was accumulated at the electrodes of opposite polarity in all the specimens within the first few seconds of polarization $(4 \sim 10 \mathrm{~s})$. This phenomenon is associated with the injection of charge pulses at one electrode, which then cross the bulk insulation at high speed and accumulate at the counterelectrode interface due to a delay in charge extraction.

The existence of ultra fast space charge pulses is evidenced in specimen no. 0 (the pure Epoxy resin) by the evolution of charge profiles, shown in Figure 3, Figures 3(a)$3(\mathrm{c})$ for the positive charge and Figures $3(\mathrm{~d})-3(\mathrm{f})$ for the negative charge. The charge pulse (packet) is labeled by a circle. At time $t=0 \mathrm{~s}$, the charge pulse appears near one electrode and begins to move towards the counterelectrode. The relative time is indicated on the top of each profile to highlight the transport velocity of each charge packet. It can be seen that positive charge pulses move slower than negative pulses. In pure epoxy resin it takes about $0.08 \mathrm{~s}$ and $0.06 \mathrm{~s}$ to cross through the bulk insulation ( $1 \mathrm{~mm}$ thick) for positive and negative fast charge packet respectively. It can be seen also that the shape of the charge pulse does not change appreciable, as a function of traveling time. Ultra fast space charge profiles obtained under the same measurement conditions for specimens nos. 1, 5, 10, and 20 containing nanoparticles are depicted in Figures 4 to 7 . All the fast charge pulses have similar features to those detected in pure epoxy resin, that is, the shape of the charge profile does not change and the pulse mobility remains constant during transit of the bulk insulation. However, the transit 


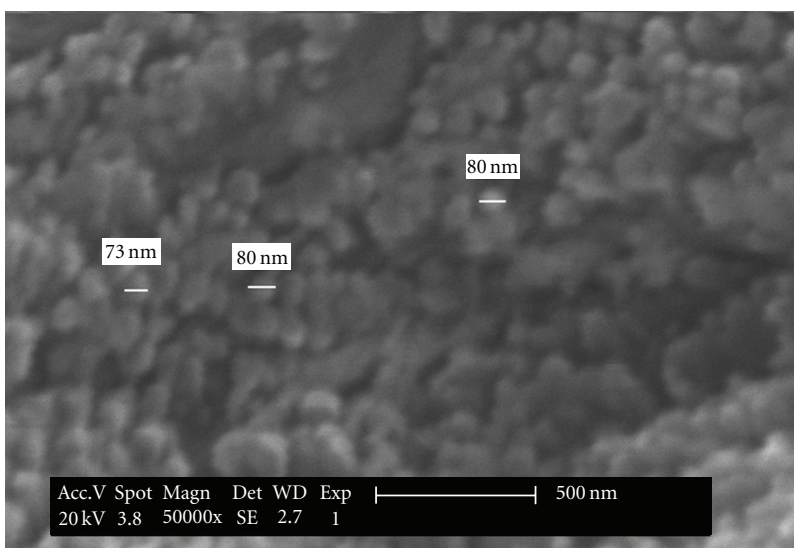

FIGURE 1: SEM image of nanocomposite (specimen no. 10, $\times 50000)$.

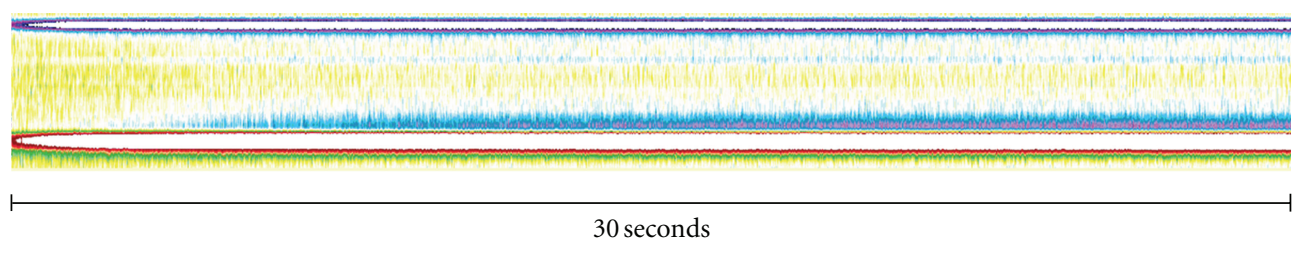

$3 e+0$

$2 e+0$

$1 e+0$

$0 e+0$

$-1 e+0$

$-2 e+0$

$-3 e+0$

(a)

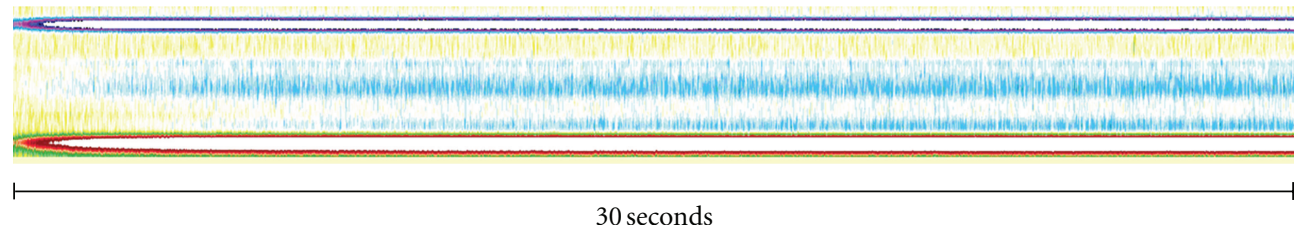

$3 e+0$

$2 e+0$

$1 e+0$

$0 e+0$

$-1 e+0$
$-2 e+0$

$-3 e+0$

(b)

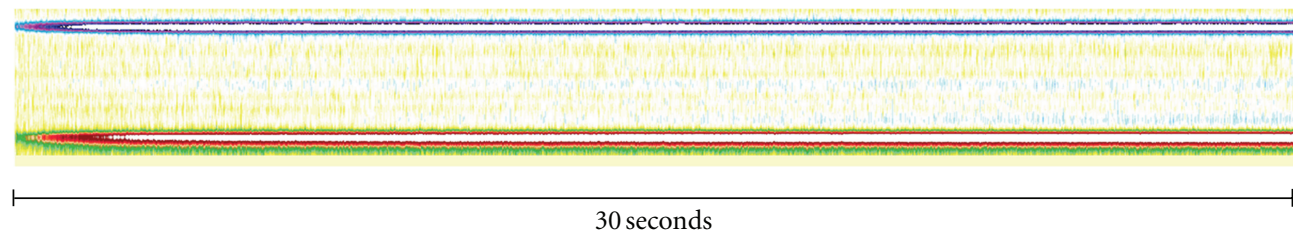

$3 e+0$

$2 e+0$

$1 e+0$

$0 e+0$

$-1 e+0$

$-2 e+0$

30 seconds

$-3 e+0$

(c)

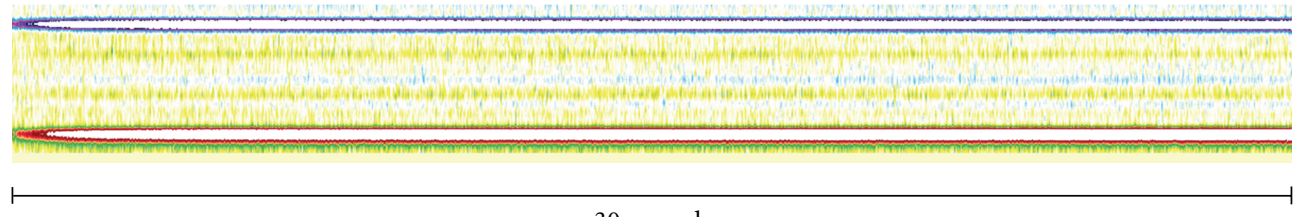

$3 e+0$

$2 e+0$

$1 e+0$

$0 e+0$

$-1 e+0$

$-2 e+0$

30 seconds

$-3 e+0$

(d)

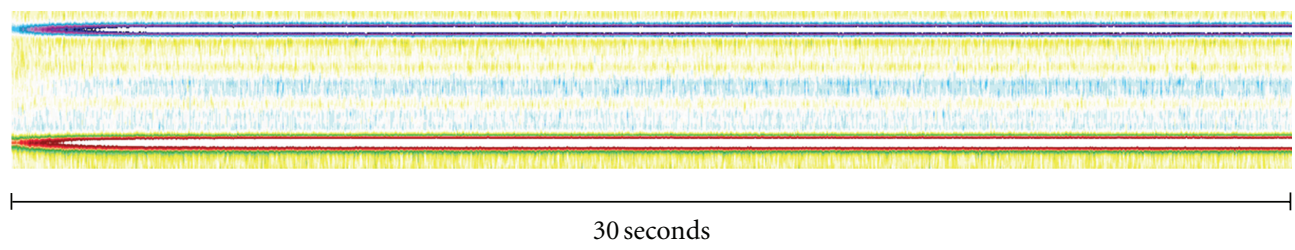

$3 e+0$

$2 e+0$

$1 e+0$

$0 e+0$

$-1 e+0$

$-3 e+0$

(e)

Figure 2: Charge patterns of specimens in the first 30 seconds of polarization. (a) No. 0, (b) no. 1, (c) no. 5, (d) no. 10, and (e) no. 20. 


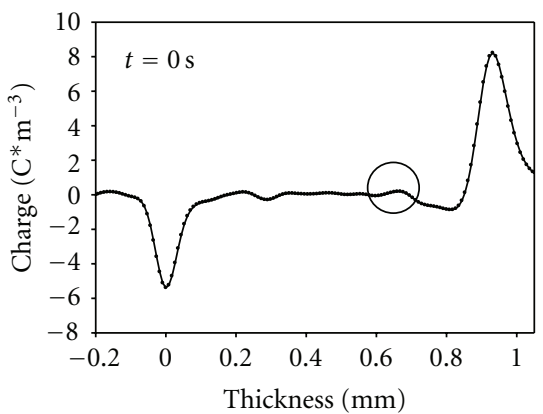

(a)

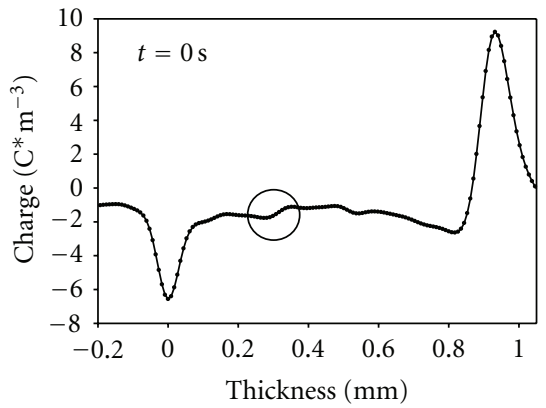

(d)

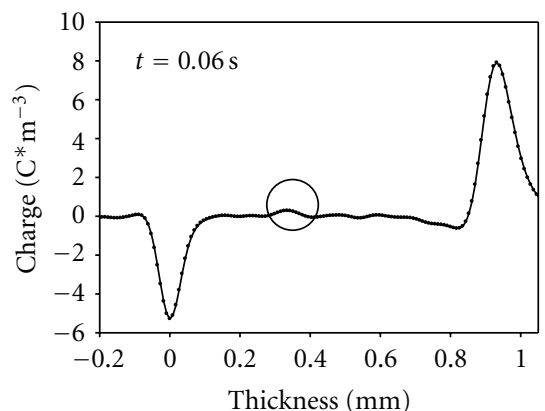

(b)

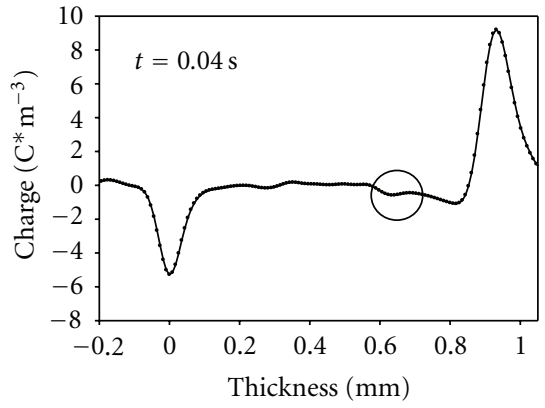

(e)

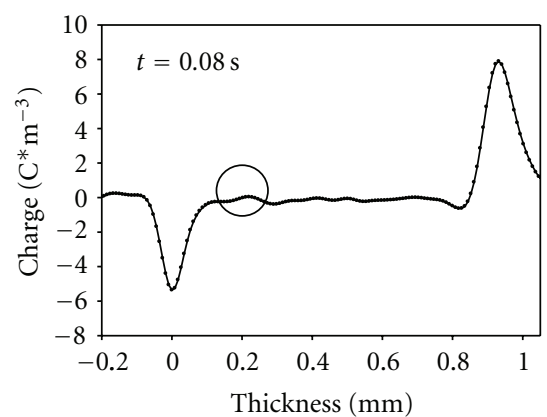

(c)

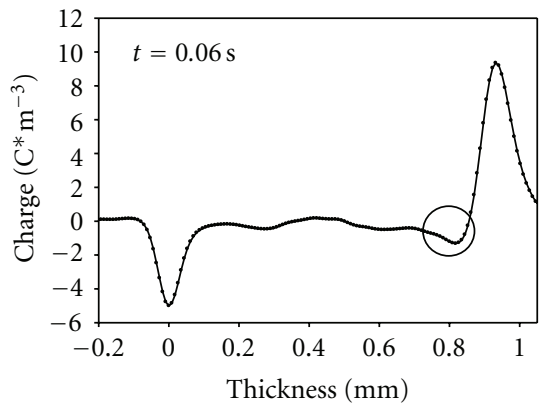

(f)

FIGURE 3: Evolution of charge profiles in specimen no. 0 under an electrical field of $10 \mathrm{kV} / \mathrm{mm}$ and temperature of $70^{\circ} \mathrm{C}$ for a positive charge pulse (a)-(c) and negative charge pulse (d)-(f).

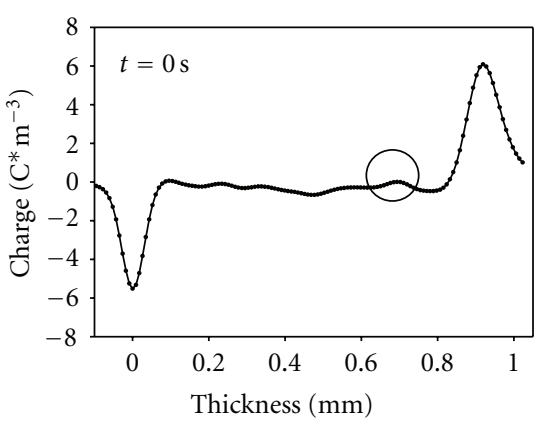

(a)

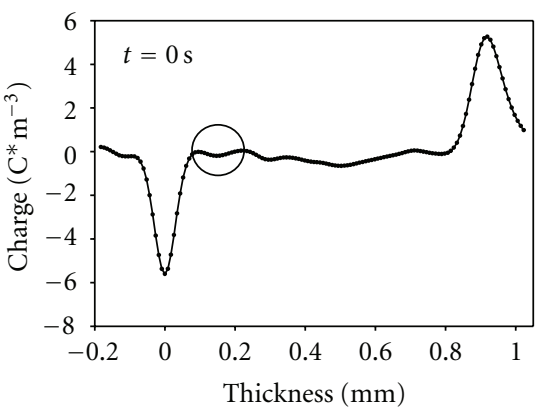

(d)

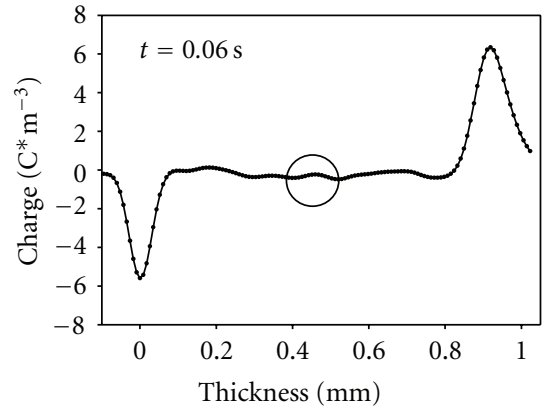

(b)

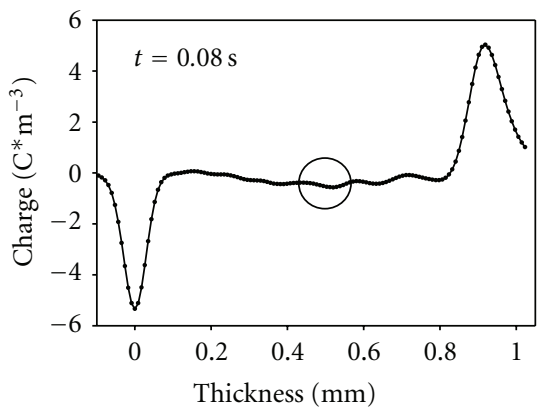

(e)

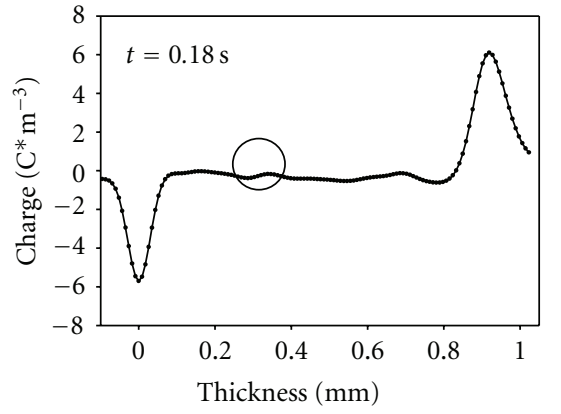

(c)

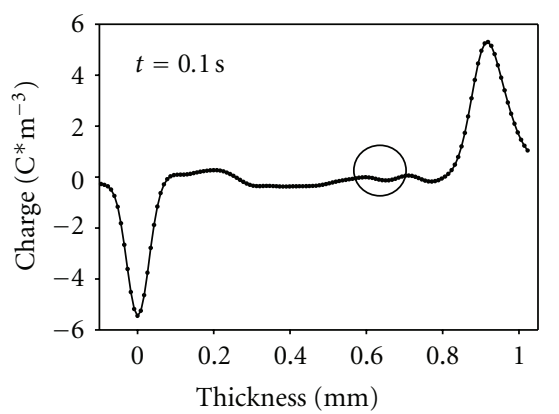

(f)

FIGURE 4: Evolution of charge profiles in specimen no. 1 under an electrical field of $10 \mathrm{kV} / \mathrm{mm}$ and temperature of $70^{\circ} \mathrm{C}$ for a positive charge pulse (a)-(c) and a negative charge pulse (d)-(f). 


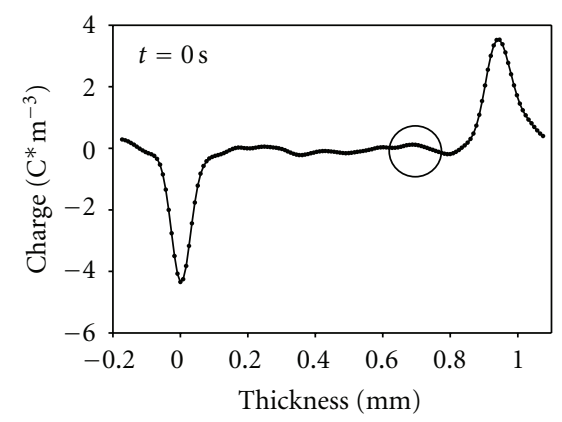

(a)

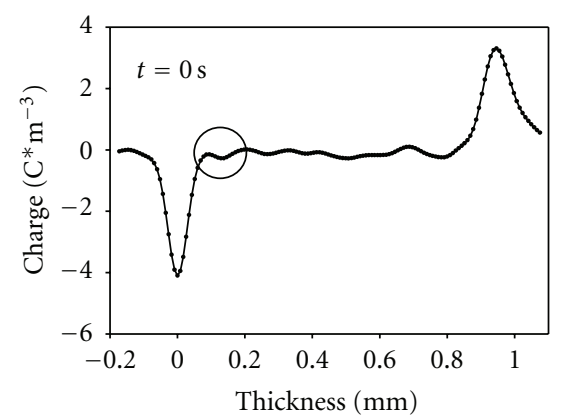

(d)

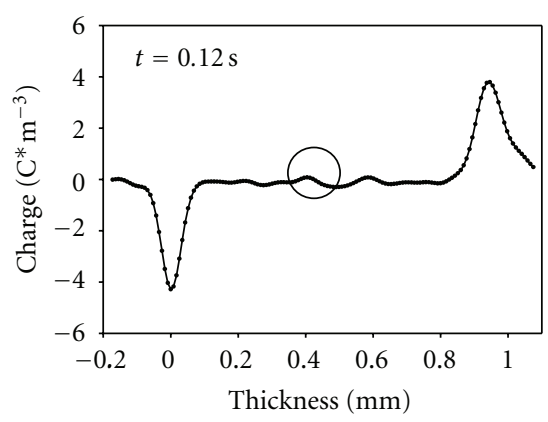

(b)

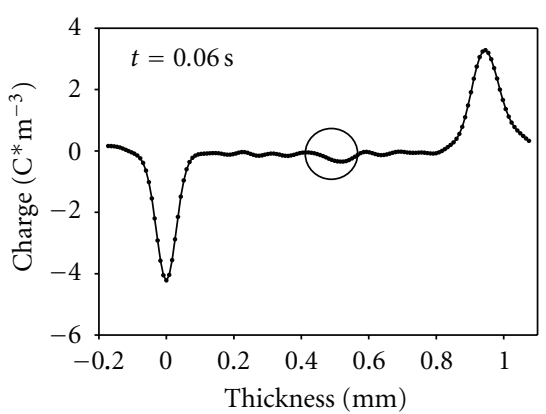

(e)

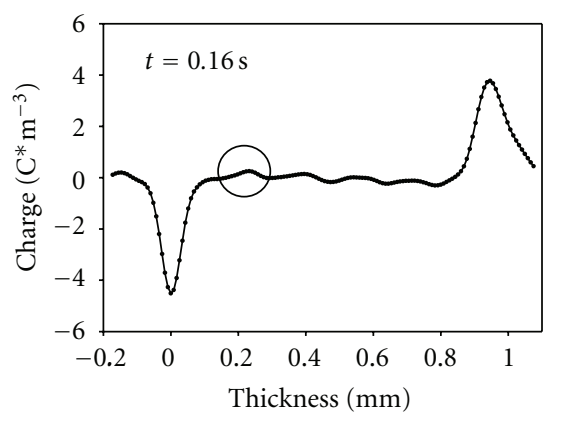

(c)

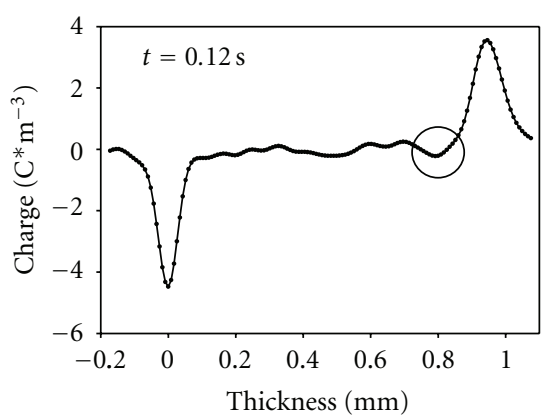

(f)

FIGURE 5: Evolution of charge profiles in specimen no. 5 under an electrical field of $10 \mathrm{kV} / \mathrm{mm}$ and temperature of $70^{\circ} \mathrm{C}$ for a positive charge pulse (a)-(c) and a negative charge pulse (d)-(f).

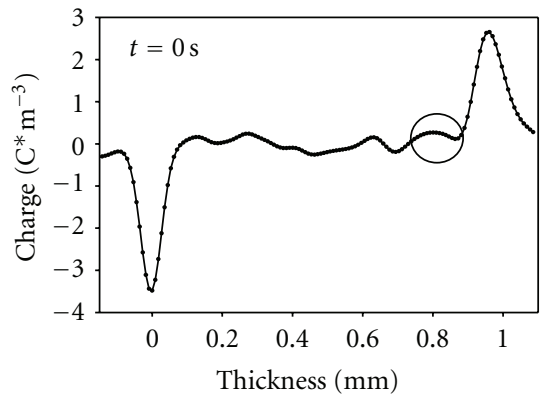

(a)

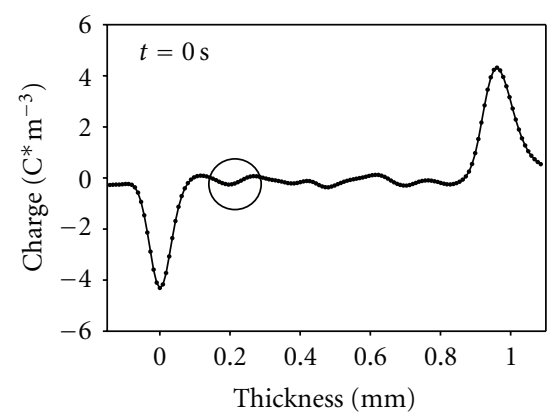

(d)

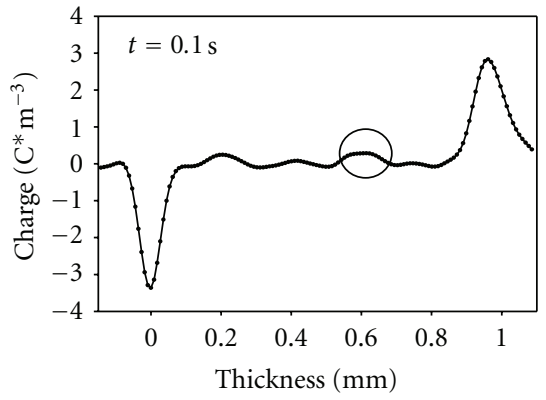

(b)

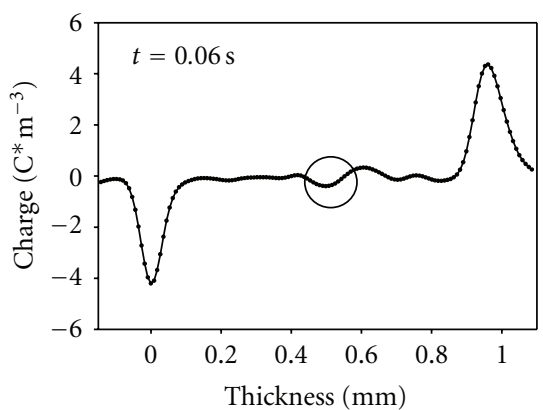

(e)

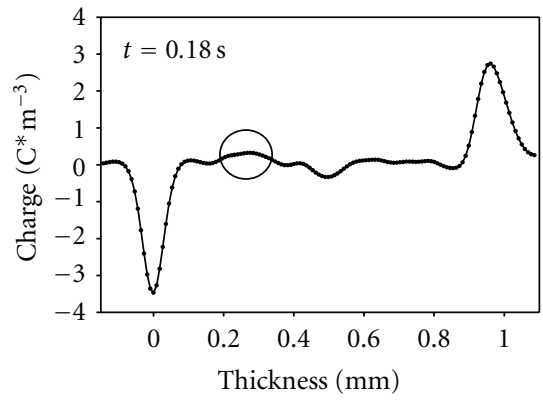

(c)

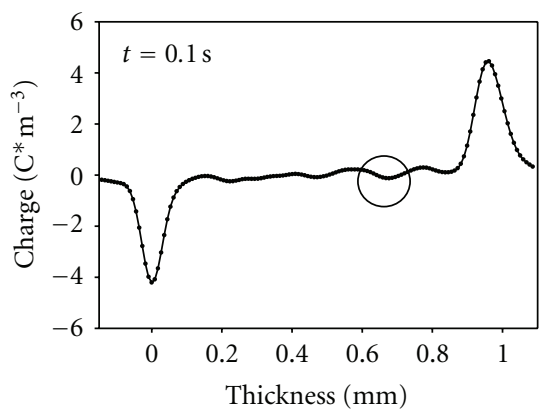

(f)

FIGURE 6: Evolution of charge profiles in specimen no. 10 under an electrical field of $10 \mathrm{kV} / \mathrm{mm}$ and temperature of $70^{\circ} \mathrm{C}$ for a positive charge pulse (a)-(c) and a negative charge pulse (d)-(f). 


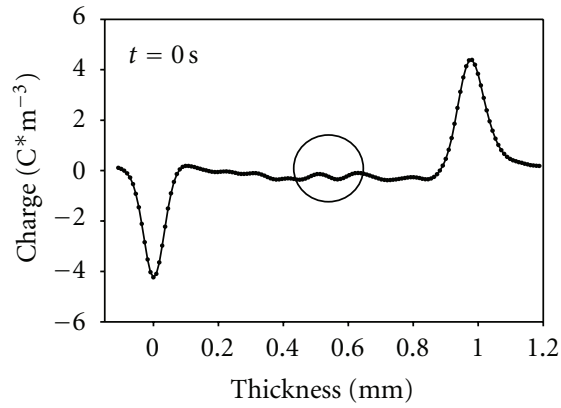

(a)

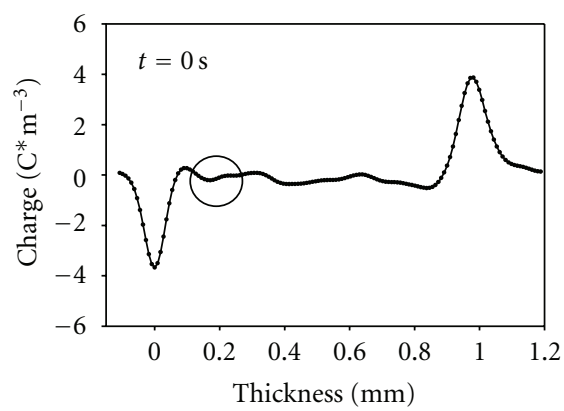

(d)

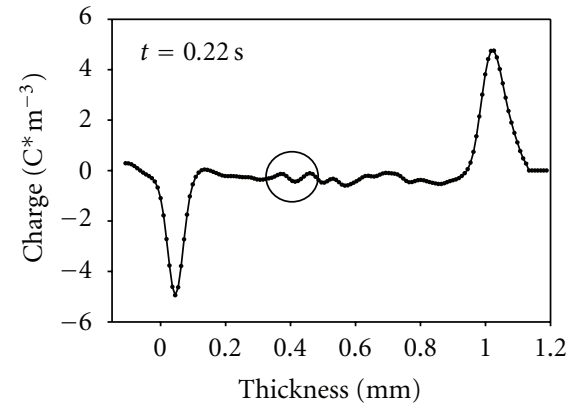

(b)

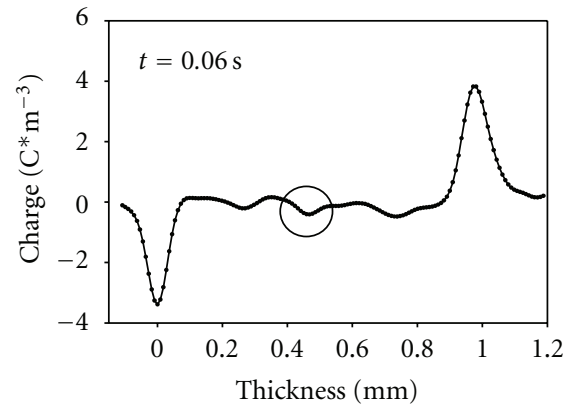

(e)

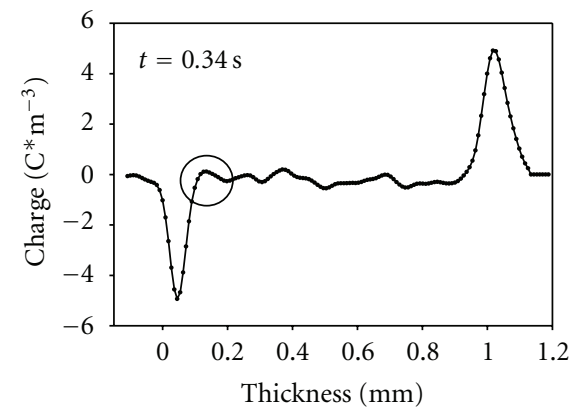

(c)

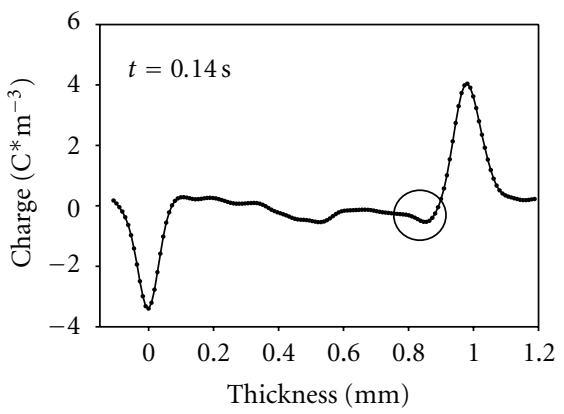

(f)

FIgURE 7: Evolution of charge profiles in specimen no. 20 under an electrical field of $10 \mathrm{kV} / \mathrm{mm}$ and temperature of $70^{\circ} \mathrm{C}$ for a positive charge pulse (a)-(c) and a negative charge pulse (d)-(f).

time in nanocomposites is longer than that in pure epoxy resin. In particular, it takes $0.34 \mathrm{~s}$ and $0.14 \mathrm{~s}$ for positive and negative charge pulses to cross the material containing $20 \%$ nanoparticles compared to the $0.08 \mathrm{~s}$ and $0.06 \mathrm{~s}$ of the pure epoxy. These figures show clearly that the transport of positive and negative charge pulses is affected significantly by the presence of nanofillers.

The injection and transport of soliton-like charge pulses in pure epoxy resin can be explained by considering the effect of the electromechanical compression at the electrodeinsulation interface. Figure 8 shows a sketch explaining such charge injection mechanism. According to Lewis et al. [12], the applied electric field produces a precompression at the electrode-polymer interface. A critical amount of charge, $\Delta Q$, needs to be available at the electrode-insulation interface in order to create an extracompression of the polymer chains against the electrode suitable for the charge $\Delta Q$ to tunnel into the chain in the form of a charge layer. After charge injection, the electric field decreases by $\Delta E$, leading the polymer chain to release, thus stopping further charge injection while simultaneously carrying the layer of charge into the polymer. Charge pulse transport occurs through the polymer chain displacements produced by electromechanical compression and electrical polarization at the charged boundary $\Delta Q, \Delta E$, which bring polymer chains close enough to allow for charge tunneling or the opening up of free space [13], thereby allowing the coherent advance of the charge layer. This mechanism allows the charge to be injected as pulses of magnitude $\Delta Q$ and transported in the bulk insulation $[7,8,13]$ as a coherent unit that is, a charge soliton.
The results obtained here for the pure epoxy resin are different to those reported previously dealing with ultra fast space charge transport in nanostructured epoxy-based materials [7]. In [7], ultra fast charge pulses could not be found in the pure epoxy material unless an external compression was applied. To understand the difference in behavior, two aspects about the materials must be highlighted. On one hand, the main chemical structures (shown in Figure 8) of the two kinds of base epoxy resin are different. The epoxy studied in the former paper is Aldrich cycloaliphatic-epoxy. The typical chemical structure is summarized in Figure 9(a). This is a kind of epoxy resin with short molecular chain and rigid groups in the main chain, such as the ring structure

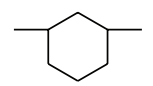

and ester bond (-COO-). The glass transition temperature $T_{\mathrm{g}}$ is very high (usually higher than $200^{\circ} \mathrm{C}[14]$ ). Hence, molecular chains cannot move easily for lack of flexibility, that is, it is difficult to compress the polymer as required for the charge soliton mechanism. In contrast, the epoxy resin studied here is a bisphenol A epoxy, see Figure 9(b), which has longer polymer chains. There are several different kinds of flexible bonds in the main chain, such as carboncarbon bonds $(\mathrm{C}-\mathrm{C})$ and ether bonds $(-\mathrm{O}-)$, which will provide more flexibility to the molecular chain. In addition, the curing procedures of the two epoxy-based materials are different. The epoxy resin studied previously is solidified by means of radiation without hardener, according to which, 


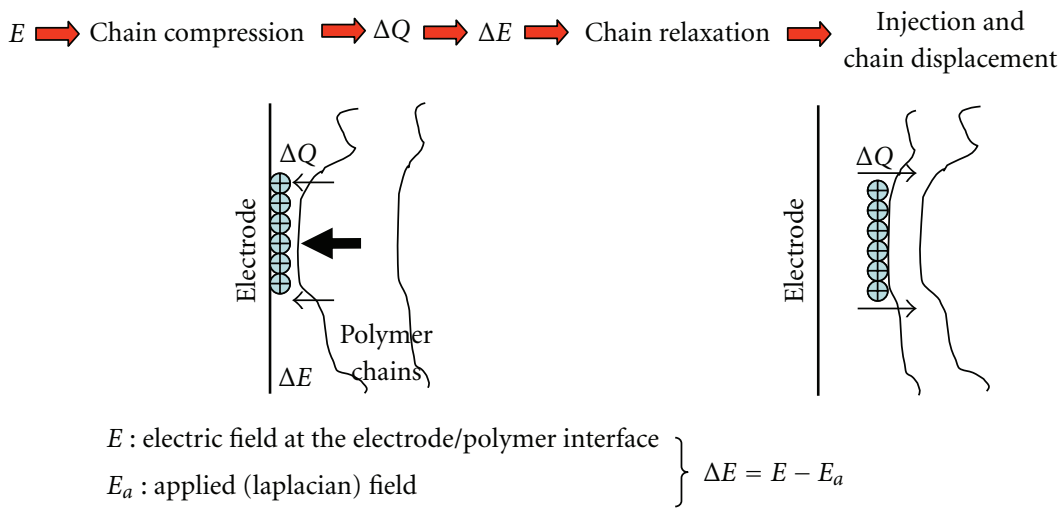

FIGURE 8: Soliton injection mechanism due to electromechanical compression of the electrode-insulation interface.<smiles>CC1CC2OC2CC1COC(=O)C1CC2OC2CC1C</smiles>

(a)<smiles>CC1OC1COc1ccc(C(C)(C)c2ccc(OCC(O)COc3ccc(C(C)(C)c4ccc(OCC5CO5)cc4)cc3)cc2)cc1</smiles>

(b)

FIgURE 9: Chemical structures of two kinds of epoxy resin (a) cycloaliphatic-epoxy, (b) bisphenol A epoxy.

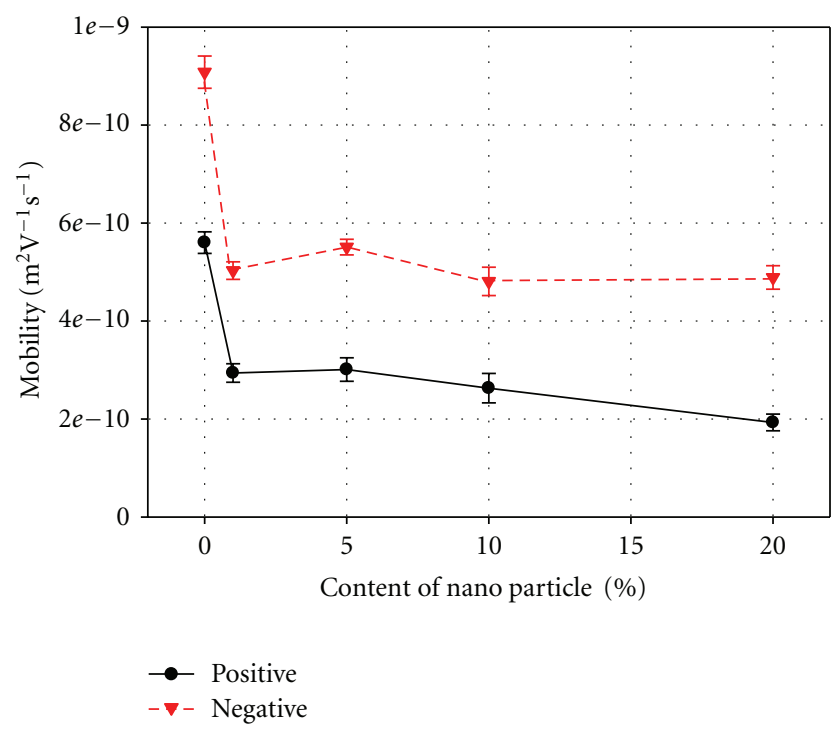

Figure 10: Charge mobility of positive and negative charge pulses in pure epoxy resin and epoxy-based nanocomposites $(90 \%$ confidence interval). the solidification of epoxy resin involved the ring-opening reaction at the epoxide groups<smiles>CC1CO1</smiles>

of chains, cross-linked with each other. Therefore, the structure of the epoxy is tight and polymer chains are close to each other. In the specimen investigated here, the epoxy hardening system consists of hardener (Anhydride) and $20 \mathrm{wt} \%$ flexibilizer. The epoxy chains are connected by small molecules. It is because of the presence of so many small molecules dispersed in the polymer chains that there is enough free volume for chains to move and change position, so that the $T_{g}$ of this kind of epoxy resin is rather low (about $20^{\circ} \mathrm{C}$ ). As mentioned above [13], it has been proposed that the generation and transport of ultra fast charge pulses in polymers is related to electromechanical compression and movement of polymer chains undergoing the corresponding relaxation process (e.g., $\beta$ and $\gamma$ relaxation in polyethylene $[5,6,8])$. Hence, the more flexible the molecular chain is, the easier ultra fast charge pulses are generated. 


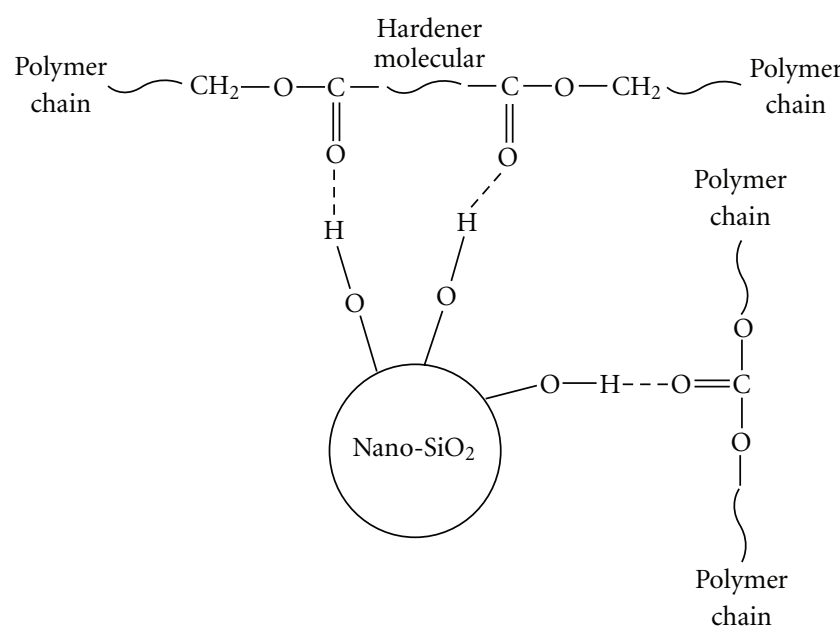

(a)

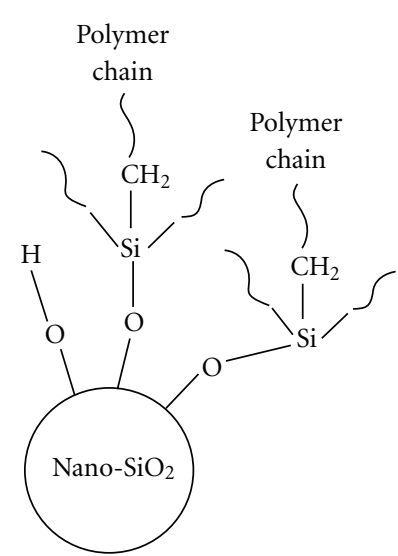

(b)

FIGURE 11: Cross-link structure built by nanoparticle in polymer chain (a) crosslinked by physical absorption (H-bond) (b) crosslinked by chemical bond (Coupling agent).

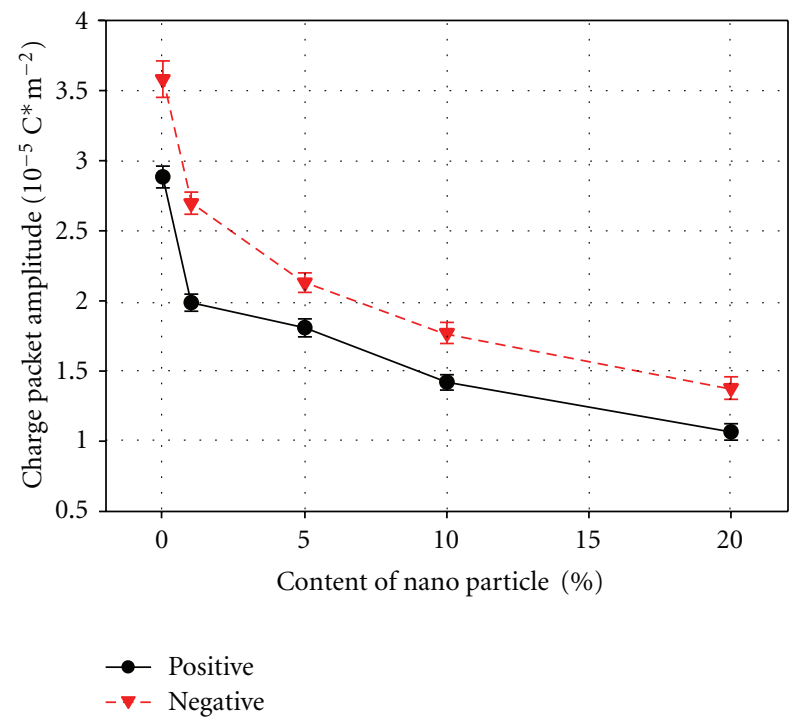

FIGURE 12: Charge pulse amplitude for pure epoxy resin and epoxybased nanocomposites ( $90 \%$ confidence interval).

The different behavior of the two pure epoxy specimens, that is, cycloaliphatic and bisphenol, the former in its glassy state and the latter in its flexible state, lend support to the proposed conduction mechanism in which electromechanical compression, which may be influenced by the structure of polymer, controls the ultra fast charge pulse conduction.

The mobility $(\mu)$ of charge packet can be calculated from

$$
\mu=\frac{v}{E}
$$

where $v$ is charge velocity $\left(\mathrm{ms}^{-1}\right)$ and $E$ is the average electrical field $\left(\mathrm{Vm}^{-1}\right)$.

An estimate of the velocity of a charge packet, $v$, can be obtained from the charge profiles (e.g., Figures 3-7) and used to calculate the mobility of both positive and negative charge pulses in specimens acquired under the condition of $10 \mathrm{kV} \mathrm{mm}^{-1}$ and $70^{\circ} \mathrm{C}$, see Figure 10 . It can be seen that the mobility of the ultra fast charge pulses is in the range of $10^{-9}-10^{-10} \mathrm{~m}^{2} \mathrm{~V}^{-1} \mathrm{~s}^{-1}$, which is much higher than conventional charge mobility. In particular, the mobility is $5.6 \times 10^{-10} \mathrm{~m}^{2} \mathrm{~V}^{-1} \mathrm{~s}^{-1}$ for positive charge pulses and 9.1 $\times 10^{-10} \mathrm{~m}^{2} \mathrm{~V}^{-1} \mathrm{~s}^{-1}$ for negative charge pulses, respectively, in the pure epoxy resin. The introduction of nanoparticles into the base polymer causes the charge pulse mobility and amplitude to decrease, the latter to $1 / 3-1 / 4$ of its value in the pure resin. It should also be noticed that the positive charge pulse mobility decreases much more than that of negative charge pulses when increasing the nanoparticle content in the composite.

In [13] it was speculated that the positive solitons (charge pulses) move by means of the single bond rotation (e.g., $\gamma$ mode in $\mathrm{PE}$ ), which allows hole transfer to a neighboring chain by reverse tunneling of electrons, which then travel a distance by the subsequent chain displacement. In the case of negative soliton (charge pulse) motion, it was suggested that electron packets injected and trapped in free volume move into neighboring free volume through an easy path produced when the hindering chains are opened up by chain relaxation (e.g., $\beta$-mode in $\mathrm{PE}$ ). The different modes of motion of positive and negative charge pulses result in a different charge mobility and lack of superposition of positive and negative pulses. When nanoparticles are added to the base polymer, their large specific surface area and surface energy allows each particle to act as a cross-link point in the polymer by means of the physical absorption effect (H-bond) or chemical bond (e.g., silane coupling agent) created between particle surface and polymer chains. The chemical structure of crosslinking in the polymer through nanoparticles is sketched in Figure 11. Therefore, the cross-link degree of epoxy resin is increased by the presence of nanoparticles. In other words, the molecular chain movement and bond rotation in the 


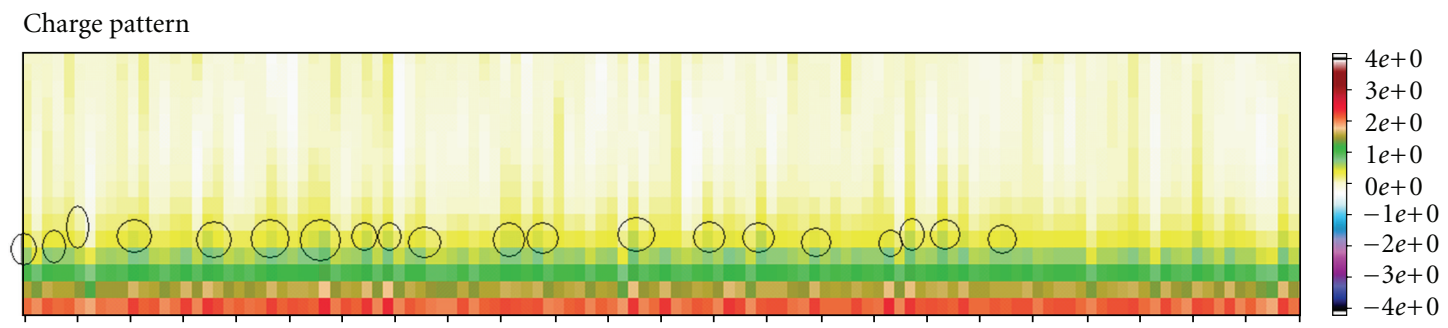

680685690695700705710715720725730735740745750755760765770775780785790795800

N. file

2 seconds

(a)

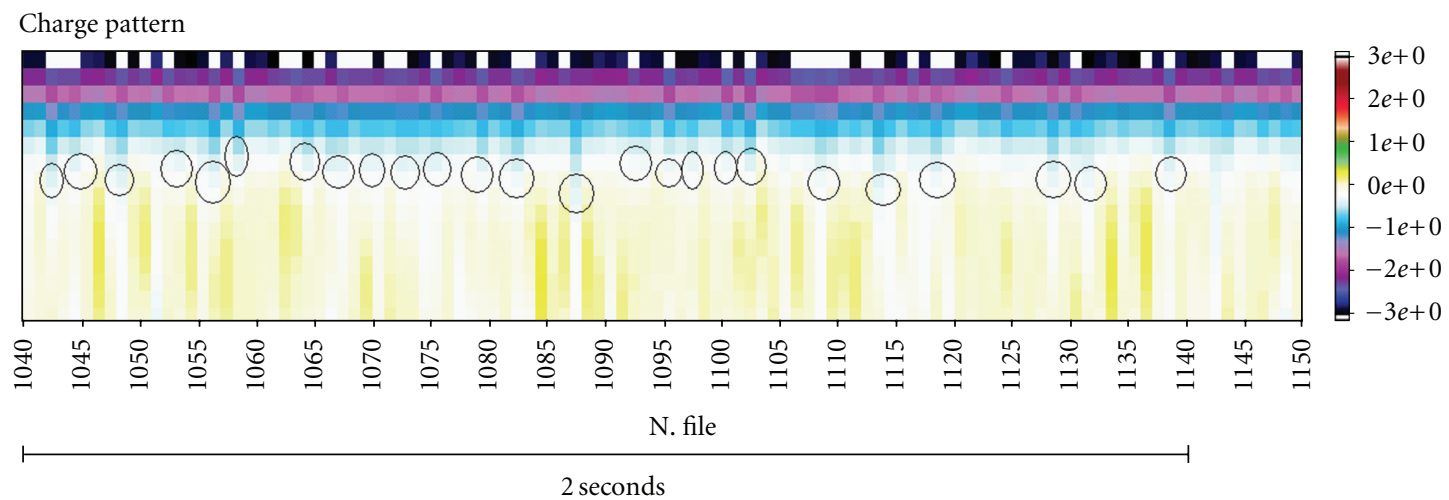

(b)

FigURE 13: Enlargement of the charge pattern in specimen no. 0 (Figure 1(a)) positive (a) and negative (b) charge pulses are labeled by circles.

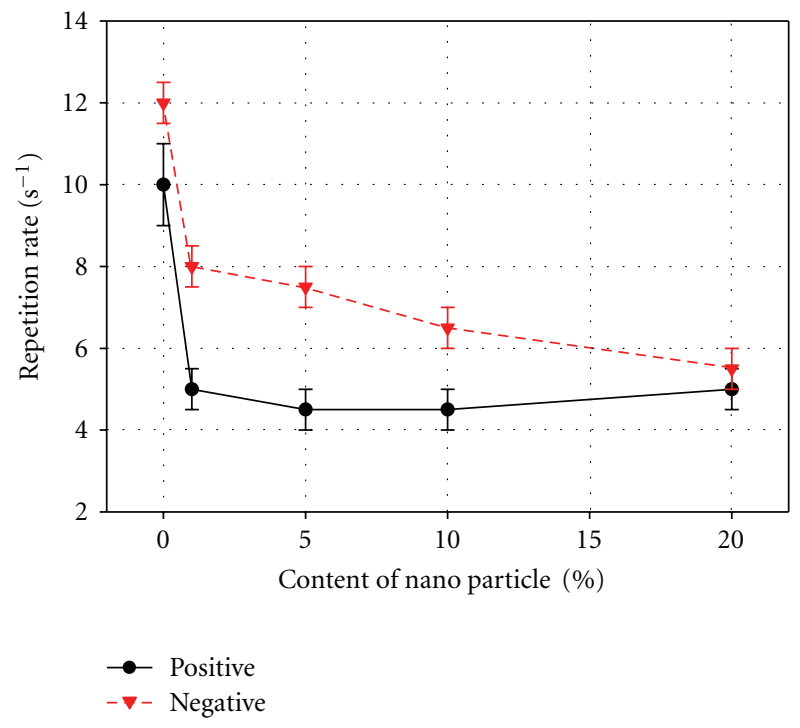

FIGURE 14: Charge pulse repetition rate of pure epoxy resin and epoxy-based nanocomposites ( $90 \%$ confidence interval).

polymer are all restricted by the presence of nanoparticles. In this circumstance, it is more difficult, for example, for positive charge pulses to tunnel into other chains or negative charge pulses to move into neighboring free volume. Consequently the mobility of both positive and negative charge pulses should decrease as observed.
The charge pulse amplitude $\left(\mathrm{Cm}^{-2}\right)$ is obtained as the area of the pulse shown in Figure 3 to Figure 7, that is, it is calculated as the integral of the charge volume density over the pulse width. The result at $T=70^{\circ} \mathrm{C}$ and $E=10 \mathrm{kV} \mathrm{mm}^{-1}$ is plotted in Figure 12. Here it can be seen that the absolute amplitudes of positive and negative charge pulses in pure Epoxy resin are $2.88 \times 10^{-5} \mathrm{Cm}^{-2}$ and $3.58 \times 10^{-5} \mathrm{Cm}^{-2}$, respectively, and that both positive and negative pulse amplitudes decrease with rising nanoparticle concentration. In particular, the amplitude in the nanocomposite with $20 \%$ nanoparticles is only $37.0 \%$ and $38.5 \%$ of that relevant to the pure material for positive and negative charge pulses respectively.

Interestingly, the influence of nanoparticles on amplitude and mobility of ultra fast charge shows a similar trend to that found for conventional (slow) high-field space charge packets. It is reported that both the amount of charge and mobility in $\mathrm{MgO} / \mathrm{LDPE}$ nanocomposite decrease with nanofiller content, which is considered that the trapping effect of the injected charge by $\mathrm{MgO}$ nanofiller may hinder the space charge injection and charge transport in polymer [15].

The number of electronic charges contained in one fast charge pulse can be calculated by multiplying the pulse charge amplitude $\left(\mathrm{Cm}^{-2}\right)$ by the area of the PEA electrode and dividing by the charge quantity of an electron $(1.6 \times$ $10^{-19} \mathrm{C}$ ). The results are listed in Table 2, which shows that there are more than $10^{15}$ charges in one pulse injected from the electrodes in the case of the pure polymer with 
TABLE 2: Number of electronic charges per fast packet.

\begin{tabular}{lcc}
\hline Specimen & \multicolumn{2}{c}{$\begin{array}{c}\text { Number of charge } /\left(\times 10^{14}\right) \\
\text { Positive charge }\end{array}$} \\
\hline No. 0 & 14.1 & 17.6 \\
No. 1 & 9.8 & 13.2 \\
No. 5 & 8.9 & 10.5 \\
No. 10 & 7.0 & 8.7 \\
No. 20 & 5.2 & 6.8 \\
\hline
\end{tabular}

the amount decreasing monotonically with the increase in concentration of nanoparticles in the composite. This shows that the number of electronic charges per pulse injected into insulating polymer is reduced by the presence of nanoparticles.

The charge repetition rate (RR) was estimated from the space charge patterns (Figure 2). As an example, Figure 13 shows an enlargement of the regions near the anode (a) and near the cathode (b) in Figure 2(a) in order to estimate the charge repetition rate (the charge pulses are labeled by circles). It can be seen clearly from Figure 13(a) that several positive and negative charge packets are injected into the insulating bulk. There are 20 positive and 24 negative charge packets injected into the material in 100 acquisition files, respectively, that is, about $2 \mathrm{~s}$ (the acquisition time is set to be $0.02 \mathrm{~s}$ for obtaining one charge profile). Hence, the charge packet repetition rate for positive $\left(\mathrm{RR}^{+}\right)$and negative charge $\left(\mathrm{RR}^{-}\right)$could be calculated as

$$
\begin{aligned}
& \mathrm{RR}^{+}=\frac{20}{100 \times 0.02}=10\left(\mathrm{~s}^{-1}\right), \\
& \mathrm{RR}^{-}=\frac{24}{100 \times 0.02}=12\left(\mathrm{~s}^{-1}\right) .
\end{aligned}
$$

Therefore, 10 positive and 12 negative fast charge packets are injected into pure polymer in one second, which is a higher rate than that observed in XLPE [6]. Figure 14 shows the repetition rates of the nanofilled composites counted in the same way as for specimen no. 0 . As can be seen the repetition rate reduces when nanoparticles are added into the material. In [13], it was suggested that the repetition rate was governed by the replenishment of the charge reservoir at the electrode-polymer interface to the level $\Delta Q$ at which it is capable of initiating pulse injection. The reduction of RR with increasing nanofiller content may, therefore, be due to the modification of the electrode-polymer interface caused by the presence of nanofiller.

It must be emphasized again that this new conduction phenomena occurs under relatively low electrical fields, that is, close to the operating field for electrical equipment, and has a fundamental interaction with the electromechanical property of the insulating polymer, thus with its structural characteristics. Therefore, it might be possible to apply it to the online diagnosis of insulation ageing in electrical equipment, as well as an indication of the proper nanofiller concentration to achieve specific electromechanical characteristics.

\section{Conclusions}

Ultra fast space charge pulses have been observed in flexible pure epoxy resin and epoxy-based nanocomposites. The characteristics of these ultra fast charge pulses, that is, mobility, amplitude, and repetition rate, are affected significantly by the concentration of nanoparticles, for both positive and negative charge pulses. Such phenomena can be explained by a new conduction mechanism associated with the chemical structure and electrical/mechanical properties of an insulating polymer, which can be modified by nanoparticles. The effects of the nanoparticles are regarded as changing the mechanical properties by increasing cross-link degree and chain stiffness. This gives further support to the contention that the mechanism of the ultra fast soliton transport is governed by the electromechanical compression of an insulating material.

\section{References}

[1] D. A. Seanor, Electrical Properties of Polymers, Academic Press, London, UK, 1982.

[2] L. A. Dissado and G. C. Fothergill, Electrical Degradation and Breakdown in Polymers, Peregrinus Press, Stevenage, UK, 1992.

[3] G. C. Montanari, "The electrical degradation threshold of polyethylene investigated by space charge and conduction current measurements," IEEE Transactions on Dielectrics and Electrical Insulation, vol. 7, no. 3, pp. 309-315, 2000.

[4] G. C. Montanari, "Dielectric material properties investigated through space charge measurements," IEEE Transactions on Dielectrics and Electrical Insulation, vol. 11, no. 1, pp. 56-64, 2004.

[5] D. Fabiani, G. C. Montanari, L. A. Dissado, C. Laurent, and G. Teyssedre, "Fast and slow charge packets in polymeric materials under DC stress," IEEE Transactions on Dielectrics and Electrical Insulation, vol. 16, no. 1, Article ID 4784573, pp. 241-250, 2009.

[6] D. Fabiani, G. C. Montanari, and L. A. Dissado, "Space charge accumulation due to ultra fast charge packets in XLPE insulated cables: the effect of temperature and field," in Proceedings of the 9th IEEE International Conference on Properties and Applications of Dielectric Materials (ICPADM '09), pp. 337340, Harbin, China, 2009.

[7] D. Fabiani, G. C. Montanari, E. Siracusano, and L. A. Dissado, "Ultra fast space charge packets in nanostructured epoxy-based materials," in Proceedings of the Annual Report Conference on Electrical Insulation and Dielectric Phenomena (CEIDP '09), pp. 31-34, West Lafayette, Ind, USA, October 2009.

[8] D. Fabiani, G. C. Montanari, and L. A. Dissado, "Fast pulselike conduction in XLPE based materials detected through charging current measurements," in Proceedings of the IEEE International Conference on Solid Dielectrics (ICSD '10), pp. 14, Postan, Germany, 2010.

[9] G. Polizos, V. Tomer, E. Manias, and C. A. Randall, "Epoxybased nanocomposites for electrical energy storage. II: nanocomposites with nanofillers of reactive montmorillonite covalently-bonded with barium titanate," Journal of Applied Physics, vol. 108, no. 7, Article ID 074117, 10 pages, 2010.

[10] T. J. Lewis, "Nanometric dielectrics," IEEE Transactions on Dielectrics and Electrical Insulation, vol. 1, no. 5, pp. 812-825, 1994. 
[11] S. Li, G. Yin, G. Chen et al., "Short-term breakdown and longterm failure in nanodielectrics: a review," IEEE Transactions on Dielectrics and Electrical Insulation, vol. 17, no. 5, pp. 1523$1535,2010$.

[12] T. J. Lewis, J. P. Llewellyn, and M. J. van der Sluijs, "Electrokinetic properties of metal-dielectric interfaces," IEEE Transactions on Dielectrics and Electrical Insulation, vol. 140, no. 5, pp. 385-392, 1993.

[13] D. Fabiani, G. C. Montanari, and L. A. Dissado, "High mobility conduction in insulating polymers through fast soliton-like charge pulses," Journal of Physics: Conference Series, vol. 183, Article ID 012010, pp. 1-6, 2009.

[14] X. Huang, Y. Zheng, P. Jiang, and YI. Yin, "Influence of nanoparticle surface treatment on the electrical properties of cycloaliphatic epoxy nanocomposites," IEEE Transactions on Dielectrics and Electrical Insulation, vol. 17, no. 2, Article ID 5448121, pp. 635-643, 2010.

[15] Y. Murakami, M. Nemoto, S. Okuzumi et al., "DC conduction and electrical breakdown of $\mathrm{MgO} / \mathrm{LDPE}$ nanocomposite," IEEE Transactions on Dielectrics and Electrical Insulation, vol. 15, no. 1, pp. 33-39, 2008. 

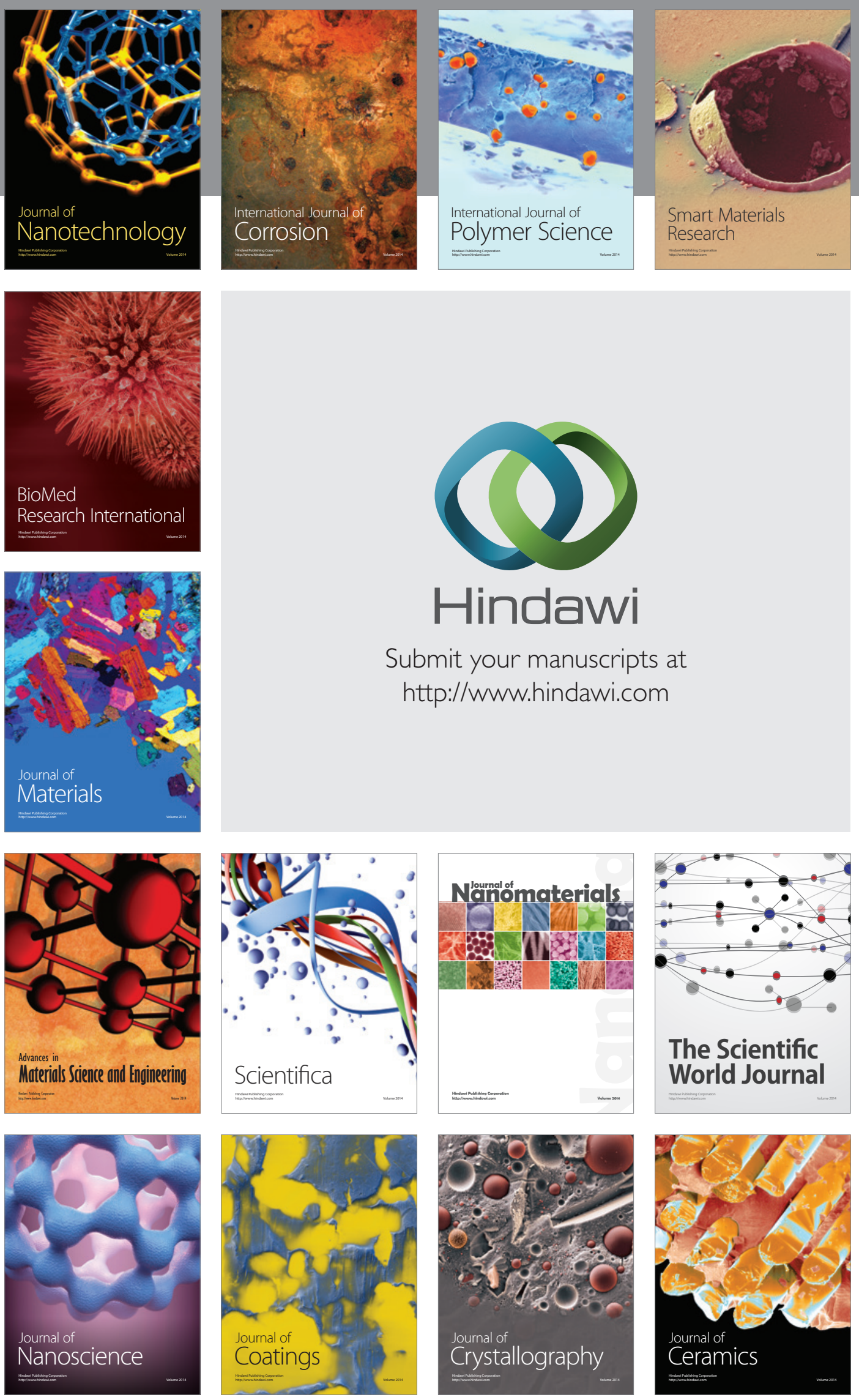

The Scientific World Journal

Submit your manuscripts at

http://www.hindawi.com

\section{World Journal}

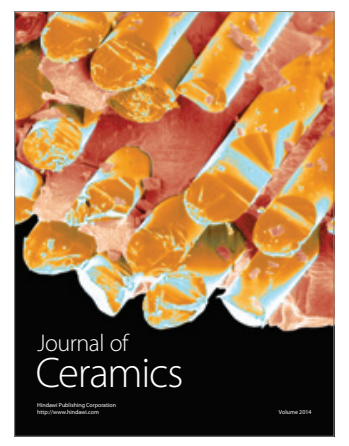

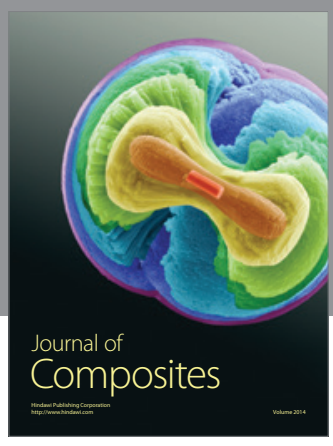
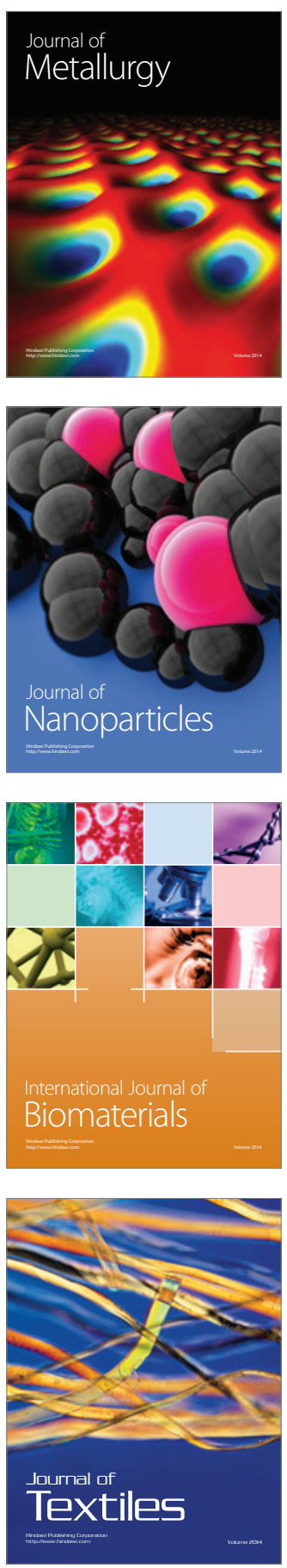